\title{
A RELAÇÃO ENTRE MOTIVAÇÃO E AUTORREGULAÇÃO DAS APRENDIZAGENS
}

\author{
Sueli Édi Rufini ${ }^{1}$ \\ Jose Aloyseo Bzuneck ${ }^{2}$
}

Resumo: No presente artigo foi exposto como a relação entre motivação e autorregulação foi considerada por teóricos na história mais recente da Psicologia Educacional. Mais especificamente, é examinada a literatura sobre constructos motivacionais que antecedem e promovem a aprendizagem autorregulada. Três modelos teóricos foram os referenciais mais adotados nas pesquisas. Primeiro, foram apresentados e discutidos resultados de estudos sobre o papel da autoeficácia para a aprendizagem autorregulada. Segundo, a Teoria das Metas de Realização inspirou diversos estudos em que foi demonstrada a influência da meta domínio sobre uso de estratégias. E, por último, alguns estudos à luz da Teoria da Autodeterminação forneceram evidências de que a qualidade motivacional faz diferença para a autorregulação. Do conjunto de resultados apurados à luz dos três modelos teóricos foram extraídas conclusões para a atuação de professores nos contextos educativos.

Palavras-chave: Aprendizagem autorregulada. Estratégias de aprendizagem. Motivação para autorregulação. Teorias motivacionais.

${ }^{1}$ Doutora em Educação pela Universidade Estadual de Campinas. Professora associada da Universidade Estadual de Londrina.

2 Pedagoga, Mestra pelo Programa de Pós-graduação em Educação da Universidade Estadual de Londrina (UEL) e Doutoranda do Programa de Pós-graduação em Educação da Faculdade de Educação da Universidade Estadual de Campinas (UNICAMP).E-mail: bzuneck@sercomtel.com.br 


\title{
RELATIONSHIP BETWEEN MOTIVATION AND SELF-REGULATED LEARNING
}

\begin{abstract}
This article shows how in recent history of Educational Psychology theorists considered relationships between motivation and self-regulation. More specifically, the aim was to review the literature about motivational constructs that antecede and further self-regulated learning. Researchers have chosen three theoretical models. First, studies results about the efficacy beliefs and their role for self-regulated learning were presented and discussed. Second, Achievement Goals Theory inspired many studies and mastery goal appeared as more often related to self-regulated learning. Last, some studies on the light of Self-Determination Theory provided evidences that motivational quality for self-regulation matters. Conclusions were drawn from those studies, and educational suggestions for teachers at school were proposed.
\end{abstract}

Keywords: Self-regulated learning. Learning strategies. Motivation for self-regulation. Motivational theories.

\section{LA RELACIÓN ENTRE MOTIVACIÓN Y AUTORREGULACIÓN DEL APRENDIZAJE}

Resumen: El artigo tuvo como foco presentar las maneras por las cuales los teóricos en la reciente historia de la Psicología Educacional consideraran las relaciones entre motivación y aprendizaje autorregulada. Más específicamente, fue presentada la literatura sobre constructos motivacionales que preceden y fomentan el aprendizaje autorregulado. Los investigadores han escogido tres modelos teóricos. Primero, fueran presentados y discutidos los resultados de investigaciones sobre las creencias de autoeficacia y su relación con aprendizaje autorregulado. Segundo, la Teoría de Metas de Realización inspiró muchos estudios y la meta de logro surgió más frecuentemente como asociada con aprendizaje autorregulado. Finalmente, algunas investigaciones con el enfoque de la Teoría de Autodeterminación proporcionaron evidencias sobre la relevancia de la calidad de la motivación. De los estudios fueran extraídas conclusiones, así como sugestiones para profesores en el contexto educacional.

Palabras clave: Autorregulación del aprendizaje. Estrategias de aprendizaje. Motivación para autorregulación. Teorías motivacionales. 



\section{A relação entre motivação e autorregulação das aprendizagens}

Na história da Psicologia Educacional, ocorreu notável mudança de perspectiva nos estudos sobre o uso de estratégias de aprendizagem e a motivação. Segundo Pintrich (1989), até cerca da metade dos anos 1980, os psicólogos educacionais se dividiam em dois grupos independentes. Um grupo se dedicava aos processos de aprendizagem, bem como às estratégias exigidas para se obterem os resultados desejáveis. São citados três exemplos dessa linha cognitivista de trabalho. 0 primeiro consta dos estudos experimentais sobre memória, atenção e codificação. 0 segundo exemplo consistiu nos estudos sobre aprendizagem por descoberta. E, por último, são citados os estudos sobre mudança conceitual, até hoje preconizados na área de ciências.

Já outro grupo de pesquisadores estudava a motivação, porém sem aludir aos processos cognitivos de aprendizagem trabalhados pelos autores do primeiro grupo. Como consequência, a ocorrência de estudos conduzidos paralelamente e sem qualquer integração refletia-se nos manuais, na formação em psicologia educacional, culminando provavelmente na prática em sala de aula, ou seja, os professores recebiam uma orientação para trabalharem ora um aspecto, ora outro, mas sem os integrarem.

Ainda de acordo com Pintrich (1989; ver também MCCOMBS, 2017), a partir do final dos anos 1980, já aparecia bem definida a tendência de os estudiosos considerarem a interação dinâmica entre estratégias, como componentes cognitivos, e a motivação, ou seja, surgiu a tendência de se associar o estudo de estratégias de autorregulação e a diversidade de constructos teóricos que formam a psicologia motivacional. Assim, podese concluir que a interação dinâmica entre processos motivacionais e processos cognitivos representados pelo uso de estratégias significa, metaforicamente, uma feliz combinação do "quente" com o "frio", do poético com o matemático, do fervor com a medida, da paixão com a exatidão (PINTRICH, 1989). A metáfora do aspecto "quente" da motivação em relação com aprendizagem autorregulada foi também usada por Zusho (2017).

Dessa forma, como observaram Schunk e Zimmerman (2008), a pesquisa sobre aprendizagem autorregulada tem focalizado simultaneamente sua relação com motivação dos alunos. Entretanto, essa relação tem sido estudada sob aspectos 
diferentes. Alguns modelos de Aprendizagem Autorregulada (como desenvolvidos por autores como BOEKAERTS, PINTRICH; ZEIDNER, 2000; PINTRICH, 2004; ZIMMERMAN, 2013; ZUSHO, 2017) já incluem motivação, bem como uso de estratégias, ou seja, segundo esses modelos, a regulação incidirá sobre cognições, motivação e afetividade, comportamento e contexto. Com esse enfoque, Dörrenbächer e Perels (2016) e Liu et al. (2014) investigaram alunos de quatro diferentes perfis de autorregulação, juntamente com variáveis motivacionais, para avaliar a relação de cada composição com desempenho, esforço aplicado, satisfação e ansiedade de provas, entre outros efeitos. Já a autorregulação da própria motivação e das emoções foi objeto de vários estudos, descritos em detalhe por Bzuneck e Boruchovitch (2016) e Bzuneck (2018).

Por outro lado, mais especificamente, alguns teóricos focalizaram o aspecto de que a motivação precede e promove o uso de estratégias e a aprendizagem autorregulada (BOEKAERTS; PINTRICH; ZEIDNER, 2000; PINTRICH, 2000; ZIMMERMAN, 2000, 2008). Como explicação dessa linha de associação, o uso de estratégias autorreguladas supõe esforço e dedicação de tempo por parte dos alunos, o que depende de processos motivacionais adaptadores. Dessa forma, quando alunos não veem o valor, significado ou importância do uso de estratégias, ou não tenham interesse, não se pode prever que nelas se engajem, mesmo que as conheçam.

Como último aspecto da relação citada, a motivação é considerada recorrente, no sentido de que ela mesma é alimentada pelas experiências de êxito que são, normalmente, produto do uso de estratégias eficazes aplicadas na perseguição das metas. Esse aspecto foi apontado, por exemplo, por Pintrich (1989) e por Schunk, Meece e Pintrich (2014). Como exemplo, Baars e Wijnia (2018) investigaram o efeito de um treinamento em habilidades de autorregulação sobre o desenvolvimento do constructo motivacional de autoeficácia, avaliada no cumprimento de tarefas diversas de biologia.

Quando se considera motivação no contexto escolar, como constructo que responde pelo início de uma ação e perseverança em direção a metas (SCHUNK; MEECE; PINTRICH, 2014), é preciso ter presente que não se trata de uma variável simples e unitária. Ao contrário, a literatura tem demonstrado que é multifatorial. No aluno, estar motivado é uma condição psicológica energizante, porém, derivada e promovida por diversos fatores, que podem atuar eficazmente tanto de forma isolada como em 
interação. Considerem-se algumas questões que representam diversos fatores da motivação. Qual é o objetivo ou meta que um aluno tem em mente atingir com seus estudos? Acredita ter capacidade suficiente para executar as ações exigidas nessa tarefa? Quais são as chances de conseguir êxito? Vale a pena envolver-se com tal aprendizagem? Exerce aquela atividade por pura obrigação, ou porque tem interesse pessoal?

Em ligação com essas questões, Pintrich (2003) identificou e descreveu cinco famílias básicas de constructos sociocognitivos que têm sido o foco das pesquisas recentes sobre motivação do aluno. São eles: autoeficácia adaptadora e percepções de competência; atribuições adaptadoras e crenças de controle; altos níveis de interesse e de motivação intrínseca; altos níveis de valorização; e, por último, metas que motivam e direcionam os alunos.

\section{0 presente texto}

No presente artigo, foi apresentada e discutida uma amostra de estudos mais recentes que se dedicaram a examinar o segundo aspecto acima relatado, ou seja, que a motivação precede e afeta a autorregulação estratégica. Cabe, porém, esclarecer que, historicamente, estratégias foram por primeiro investigadas sem ligação com qualquer modelo de aprendizagem autorregulada. Atualmente, há uma predominância de estudos sobre estratégias em associação com algum dos seis modelos expostos recentemente por Panadero (2017) e, por outro lado, com a perspectiva denominada Students Approach to Learning - SAL (MCCOMBS, 2017; PINTRICH, 2004).

Entre os proponentes dos modelos teóricos de autorregulação, Zimmerman (2013) conceituou aprendizagem autorregulada como o grau em que os alunos são participantes ativos de seus próprios processos de aprendizagem, nos aspectos metacognitivo, motivacional e comportamental. Desta forma, os aprendizes autorregulados caracterizam-se como agentes proativos, por colocarem em prática processos específicos que incluem o uso de estratégias relacionadas com a tarefa. Isto

é, sem conhecimento e uso de tais estratégias, não poderá haver autorregulação da aprendizagem por parte de nenhum aluno. Estratégias pertencem à categoria de 
conhecimento processual, isto é, representam o "saber como fazer", como estudar, em suma, como aprender. No contexto educativo, elas consistem do "emprego de cognição, metacognição, motivação, afeto e comportamento, com o objetivo de aumentar a probabilidade de sucesso nas aprendizagens, por possibilitar a criação de memórias significativas e recuperáveis e, por fim, o desempenho em tarefas cognitivas de ordem superior" (WEINSTEIN; ACEE; JUNG, 2011, p. 45). Assim, a presente revisão narrativa da literatura sobre a relação entre estratégias e motivação revelará estratégias normalmente ligadas aos modelos de aprendizagem autorregulada e, eventualmente, independentes de qualquer modelo, sendo revelado, em qualquer caso, o seu papel crítico para toda aprendizagem.

Entre os estudos em que se buscaram examinar a relação entre estratégias e motivação, além do de Berger e Karabelnik (2011) em que foi utilizado como referencial a teoria expectativa-valor, os autores têm dado preferência a três constructos: as crenças de autoeficácia, a orientação a metas de realização e as diversas modalidades de motivação qualitativa sistematizadas na Teoria da Autodeterminação. Na sequência, serão relatados e discutidos os resultados de uma amostra de estudos conduzidos à luz dessas três abordagens teóricas.

\section{Estudos sobre o papel da autoeficácia}

Bandura, que introduziu o constructo de autoeficácia na literatura psicológica em ligação com o conceito de agência pessoal, definiu autoeficácia como uma "crença nas próprias capacidades de executar cursos de ação exigidos para produzir determinadas realizações” (BANDURA, 1997, p. 3). As capacidades, neste contexto, adquirem o significado de inteligência, habilidades e conhecimentos e o conceito refere-se à percepção individual ou crença acerca da posse de tais capacidades. Nesse sentido, não basta que uma pessoa seja capaz de exercer certas ações, mas é imprescindível que acredite ser capaz. Essa crença possibilita à pessoa antever o futuro naquilo que é pertinente ao nível de competência e, além disso, propicia um senso de controle sobre suas ações conducentes a resultados desejáveis. 
Bandura (1997) destacou ainda que a crença nas próprias capacidades para atingir resultados desejados é poderoso fator de influência sobre a motivação para agir, para o estabelecimento de metas, a aplicação de esforço e persistência diante de dificuldades e fracassos. Em revisões da literatura estão bem documentados esses efeitos específicos da autoeficácia (por ex., BANDURA, 1997; USHER; PAJARES, 2008).

Porém, para a mensuração desse constructo, Bandura (1997) enfatizou que ele deve ser considerado sempre em relação a ações específicas, como resolver certo problema, produzir determinado texto e assim por diante. Uma área específica bem examinada em relação com autoeficácia é a autorregulação das aprendizagens (USHER; PAJARES, 2008). Crenças pessoais na capacidade de autorregulação, autoeficácia para aprendizagem autorregulada e autoeficácia autorreguladora são diferentes rótulos utilizados pelos pesquisadores interessados em investigar o uso de estratégias de autorregulação em situações de aprendizagem. Pintrich (2004) e Zimmerman (2013) são teóricos que colocaram a autoeficácia como um dos fatores motivacionais críticos em todas as fases de seus respectivos modelos de autorregulação da aprendizagem. Empiricamente, a relevância da autoeficácia percebida para a autorregulação da aprendizagem foi amplamente documentada na literatura, como se pode verificar nas revisões empreendidas, por exemplo, por Caprara et al. (2008), por Usher e Pajares (2008) e por Zimmerman (2000).

Como amostra ilustrativa da relação entre autoeficácia e uso de estratégias de aprendizagem autorregulada, o estudo clássico de Zimmerman e Martinez-Pons (1990) explorou a autoeficácia verbal e matemática de alunos do ensino fundamental. As tarefas nas duas áreas sobre as quais foi avaliada a autoeficácia eram bem específicas. Aos alunos foram apresentadas situações hipotéticas de aprendizagem e thes era solicitado que apontassem estratégias de autorregulação necessárias para os problemas apresentados. Como resultado, tanto a autoeficácia verbal como a matemática correlacionaram-se positivamente com estratégias como estabelecimento de metas e planejamento, tomada de anotações, monitoramento e autoavaliação. Houve diferenças entre alunos mais dotados e seus colegas, bem como em função da idade.

Entre as publicações mais recentes, pode citar-se o estudo de Lee, Lee e Bong (2014) com alunos do ensino médio coreanos. Foram comparados os efeitos do interesse 
individual e da autoeficácia sobre autorregulação e desempenho, em três disciplinas de seus cursos. Enquanto que interesse individual teve valor de predição direto sobre autorregulação, a autoeficácia também predizia desempenho e autorregulação, porém, com a mediação de perspectiva de notas por parte dos alunos. 0 comentário final dos autores apontou que autorregulação será mais encorajada com o fomento desses dois constructos motivacionais.

Por seu turno, Bradley, Browne e Kelley (2017) examinaram autoeficácia de universitários em relação à aprendizagem em ambiente educativo com recursos da internet. Os autores descobriram que autoeficácia para essa modalidade de aprendizagem estava intimamente associada à capacidade de os alunos regularem seu comportamento exigido nas tarefas, o que influenciou diretamente o desempenho final.

Em suma, tanto do ponto de vista teórico como empírico, o engajamento em estratégias de autorregulação das aprendizagens depende, em primeiro lugar, dessa variável motivacional específica que é a crença na própria capacidade para se autorregular. Portanto, o domínio das estratégias autorreguladoras será eficaz apenas com a condição de que o aluno acredite ou tenha a expectativa de que é capaz de colocálas em prática. Na sequência, outros constructos motivacionais serão examinados com o mesmo objetivo.

\section{Estudos sobre metas de realização}

$\mathrm{Na}$ literatura encontram-se diferentes conceituações de metas de realização. Mas, é aceito unanimemente que elas não consistem em simples objetivos comportamentais a serem atingidos (como seria resolver tantos problemas de matemática nesta manhã). Segundo Maehr e Meyer (1997), uma meta de realização consiste na percepção do propósito ou razão, um porquê de a pessoa se envolver numa atividade. Hulleman et al. (2010) definiram essas metas como representações cognitivas que orientam o comportamento para um estado final relacionado com competência, no qual a pessoa está empenhada, quer para atingir/conseguir, quer para evitar. Em suma, essas metas foram consideradas como objetivos/propósitos, ou como razões para o engajamento. 
Desde o início de seus estudos, foi identificada a meta de domínio, também denominada meta aprender, que se referia ao propósito ou objetivo de, com o cumprimento das tarefas escolares, o aluno desenvolver competência ou chegar ao domínio de uma tarefa. Também foi examinada a orientação à meta performance, que significava o propósito de demonstrar capacidade/competência, ou de ser melhor que os outros (denominada performance-aproximação), ou de não aparecer como incapaz /incompetente ou pior que os outros (performance-evitação). Mais detalhes sobre o histórico dos estudos e as consequências motivacionais dessas diferentes metas podem ser vistos em textos como de Hulleman et al. (2010) e Mouratidis et al. (2018).

Senko e Hulleman (2013) relataram estudos conduzidos desde os anos 1990, em que se examinou a relação entre orientação a uma meta de realização e o uso de estratégias de aprendizagem. Em particular, Senko e Hulleman concluíram que essas publicações, no seu conjunto, demonstravam que a orientação à meta domínio é a mais adaptadora para as aprendizagens, uma vez que com ela os alunos têm revelado mais interesse pelos conteúdos, persistência, busca de ajudas e autorregulação. Uma questão, porém, sempre tem sido levantada e que é sobre os efeitos da orientação à meta performance-aproximação, uma vez que os resultados de pesquisas têm sido conflitantes ou pouco esclarecedores. Foram brevemente descritas, a seguir, algumas pesquisas entre as mais recentes, como amostra das que adotaram o referencial da teoria de metas de realização.

No estudo de Wolters (2004) com alunos do Ensino Médio, a orientação à meta domínio apareceu associada ao emprego mais frequente de estratégias cognitivas e metacognitivas e, por outro lado, menor frequência de procrastinação nos estudos, que é uma estratégia disfuncional. Além disso, a preocupação por ser melhor que colegas, indicadora da orientação à meta performance-aproximação não foi preditora de uso de estratégias. Igualmente, o foco dos adolescentes em não aparecerem como incompetentes, ou seja, com meta performance-evitação, não tinha relação com o uso de estratégias cognitivas e metacognitivas. Novamente, esses resultados não chegaram a dirimir de vez a questão do efeito da orientação da meta performance-aproximação. Com base em sua meta-análise sobre metas de realização, Hulleman et al. (2010) sugeriram que a meta performance-aproximação, quando medida em termos de 
comparação social(ser melhor que os outros) prediz uso de estratégias de profundidade e bom desempenho. Essa relação não foi constatada quando a medida dessa meta focalizava o desejo de aparecercomo inteligente, pois, nesse caso, está relacionada com menos interesse pelas aprendizagens em si e, portanto, com despreocupação pelas estratégias.

Valle et al. (2009) identificaram quatro grupos distintos de estudantes secundaristas mexicanos, em termos de orientação a metas. Com a abordagem centrada na pessoa (para esse conceito, ver, por ex., VANSTEENKISTE; MOURATIDIS 2016), todos os alunos foram reunidos em quatro grupos em função da combinação de metas diversas a que se orientavam. Na sequência, os autores examinaram como alunos de cada grupo relataram uso de estratégias cognitivas e de autorregulação, que apareceu significativamente mais presente no grupo de alta motivação generalizada (isto é, com mescla de motivos) e no grupo de orientação à meta domínio.

Com adolescentes portugueses, Paulino, Sá e Silva (2015) encontraram correlação positiva elevada entre os escores em autorregulação da motivação e a meta de aprendizagem (outro nome para a meta domínio). Em outras palavras, a orientação à meta de aprendizagem (domínio) apareceu altamente associada com os motivos que levam os alunos a regularem a sua motivação, que foi o principal foco do estudo. Em contrapartida, surgiu correlação moderada entre autorregulação da motivação e meta de resultado-aproximação (meta performance-aproximação). Porém, foi mais alta que neste último caso a correlação com os escores na meta resultado-evitamento, um dado que foi explicado pelas autoras como possivelmente vinculado à diminuição da motivação dos estudantes no decorrer dos anos escolares e à diminuição do uso de estratégias de aprendizagem pelos adolescentes, problemas inerentes ao desenvolvimento que necessitam de mais investigações.

Ao pesquisarem uma grande amostra de estudantes peruanos do ensino médio, Matos, Vansteenkiste e Mouratidis (2017) identificaram apenas discreta evidência sobre a relação entre as metas domínio e performance-aproximação combinadas e uso de estratégias e desempenho em matemática. Por outro lado, a orientação exclusiva à meta domínio revelou-se fortemente relacionada com uso de estratégias e desempenho. 
Com a mesma abordagem centrada na pessoa adotada por Valle et al. (2009), Linnenbrink-Garcia et al. (2018), num estudo com universitários e em relação a disciplinas de ciências, identificaram quatro perfis motivacionais não apenas em função da meta de realização como também dos constructos expectativa e valor, buscando associação com uso de estratégias, entre outros efeitos. Os níveis mais altos de engajamento em estratégias foram encontrados nos perfis caracterizados por mais altos níveis de orientação à meta domínio, de competência percebida e de valorização da tarefa. Os autores argumentaram pela necessidade de se adotar mais de uma perspectiva teórica na formação dos perfis formados em função da combinação de constructos motivacionais.

No Brasil, Nascimento et al. [2018] investigou também em alunos universitários a autorregulação pelo uso de estratégias cognitivas, metacognitivas e de gerenciamento do tempo, para o cumprimento da tarefa específica de produção de um texto. Além de avaliar essas estratégias, a autora também levantou na amostra a orientação à meta domínio-aproximação e, adicionalmente, seguindo sugestão de Urdan (1997), a orientação a metas extrínsecas, como obter boa nota ou ser reconhecido. Metodologicamente, foi adotada no estudo a abordagem centrada na pessoa, com a qual se identificaram perfis distintos de alunos quanto ao uso de estratégias autorreguladas de forma combinada. Um grupo era formado por alunos altamente regulados, outro grupo era de baixa regulação e outros dois, de nível intermediário. Não houve diferença significativa entre os escores nas metas extrínsecas reveladas pelos quatro grupos. Porém, os escores na meta domínio, que é a mais adaptadora, foram significativamente mais elevados no grupo de alta regulação, em comparação com os demais. Em conclusão, a autora destacou as vantagens de se avaliar a aprendizagem autorregulada em relação a uma tarefa específica, a formação de perfis de autorregulação que combinam graus diversos de uso de estratégias e, por fim, discutiu o resultado de que melhor qualidade motivacional foi encontrada em alunos altamente regulados. 


\section{Estudos à luz da Teoria da Autodeterminação}

Pela Teoria da Autodeterminação, desenvolvida por Deci e Ryan (1985) Ryan e Deci (2017), a motivação é um conceito multidimensional, que varia em termos de qualidade e, mais especificamente, em termos de autodeterminação. Seus autores propuseram que as pessoas podem apresentar um dos tipos motivacionais, que se estendem num continuum de autorregulação motivacional, ou seja, que variam na medida em que os indivíduos livremente se envolvem numa atividade. Assim, afora o estado possível de desmotivação, configura-se a motivação controlada e, também, a motivação autônoma, que é de melhor qualidade. A primeira forma engloba a motivação extrínseca por pura regulação externa (como para obter boas notas) e a introjetada (motivação, por exemplo, para ser reconhecido), enquanto que a autônoma abarca a motivação intrínseca e a motivação extrínseca por regulação identificada, em que a aprendizagem é vista como pessoalmente importante. Outros aspectos centrais da teoria, bem como os efeitos diferenciados de cada modalidade expressa no continuum, acham-se expostos numa grande quantidade de publicações (por exemplo, GUAY et al., 2010; RYAN; DECI, 2017; VANSTEENKISTE et al., 2009).

Além disso, os alunos variam não apenas quando se consideram os escores em cada modalidade qualitativa da motivação, segundo o continuum da autorregulação, como variam em função das combinações possíveis, o que tem sido explorado em estudos com metodologia centrada na pessoa (VANSTEENKISTE; MOURATIDIS, 2016). Vansteenkiste et al. (2009), com essa metodologia, identificaram quatro perfis de alunos: os com alta motivação autônoma e baixa controlada, os com baixa motivação autônoma e alta controlada; baixos níveis em ambos os tipos; e altos níveis em ambos os tipos. A descoberta mais saliente do estudo foi que os alunos do primeiro grupo, caracterizados como de boa motivação, revelaram, quando comparados aos demais, uma adoção mais acentuada de estratégias como elaboração, pensamento crítico e gerenciamento do tempo e, por fim, apresentaram os melhores resultados de aprendizagem. Os autores apontaram, como conclusão, como a qualidade motivacional faz diferença para o processamento cognitivo e uso de estratégias de autorregulação. 
Donche et al. (2013), também com o referencial da Teoria da Autodeterminação, concentraram-se na distinção entre desmotivação, motivação controlada e autônoma, sem formação de clusters ou perfis, para examinar a relação com estratégias. Os resultados foram que a desmotivação esteve associada a ausência de regulação das aprendizagens; a motivação autônoma estava mais relacionada com uso de estratégias superficiais; já a motivação autônoma, de melhor qualidade que a controlada, apareceu positivamente associada com processamento de profundidade e estratégias de regulação.

Como representantes mais recentes da linha de pesquisas que examinaram a influência de motivação qualitativa, segundo a Teoria da Autodeterminação, Baars e Wijnia (2018), também com a metodologia centrada na pessoa, identificaram quatro clusters motivacionais, um de qualidade pobre, um de boa qualidade e outros dois intermediários. Sua metodologia, porém, incluía (a) a peculiaridade de que o exame das variáveis incidia sobre uma tarefa específica de resolver problemas de biologia e (b) uma fase de intervenção, pela qual os alunos passaram por um treinamento em autorregulação. 0 resultado mais saliente foi que, após o treinamento, surgiu relação positiva entre o perfil motivacional de melhor qualidade e a estratégia de automonitoramento durante a exceção da tarefa. Isto é, para as autoras, os alunos caracterizados com motivação autônoma prevalente tiraram mais proveito do treinamento em habilidades de resolver problemas do que os alunos com baixa motivação autônoma.

\section{Considerações finais}

O presente artigo desvendou como, na literatura, a aprendizagem autorregulada, pelo uso de estratégias de aprendizagem, foi considerada variável dependente de constructos motivacionais diversos. As crenças de autoeficácia surgiram como fator crítico, no sentido de que o engajamento em estratégias somente ocorrerá quando o aluno ou aluna acreditar ter capacidades para executar tais ações. As pesquisas desenvolvidas à luz da teoria de Metas de realização e da Teoria da Autodeterminação, respeitadas as diferenças conceituais, trouxeram em comum o dado de que, para o 
engajamento em estratégias, faz diferença a qualidade motivacional dos alunos. Não basta, portanto, que se defenda que para usar estratégias os alunos precisam estar motivados. Há motivos e razões distintas e em graus diversos, com efeitos diversificados, o que ficou mais evidente quando, no delineamento da pesquisa, o uso de estratégias foi tratado como variável dependente.

A abordagem centrada na pessoa, adotada em estudos conduzidos à luz da teoria de Metas de realização e da Teoria da Autodeterminação, tem a vantagem de permitir que os pesquisadores considerem como grupos de alunos diferem entre si não apenas numa variável mas em função da variada composição de motivos ou razões para se engajarem numa atividade de aprendizagem. Como, de fato, alunos não são geralmente movidos apenas por um motivo, a abordagem aparece com mais validade ecológica. Entretanto, mais realisticamente, alunos podem apresentar modalidades motivacionais combinadas, derivadas de diversas teorias. Como exemplos, Lee, Lee e Bong (2014) e Linnenbrink-Garcia et al. (2018) trouxeram um avanço nesse sentido, ao usarem medidas de constructos derivados de mais de uma teoria: interesse e autoeficácia, no primeiro caso, e metas de realização com a teoria expectativa-valor, no segundo caso.

Qual consequência prática pode ser extraída desses resultados, que apresentam elevado grau de consistência, ao comprovarem a relação entre constructos motivacionais e uso de estratégias de autorregulação? Sem dúvida, sem descuidar do ensino de todo o leque de estratégias (BAARS; WIJNIA, 2018; WEINSTEIN; MAYER, 1986), os três enfoques teóricos e os presentes dados apontam para a necessidade de se socializar os alunos em três direções que não são mutuamente excludentes.

Em primeiro lugar, crenças de autoeficácia especificamente talhadas para a aprendizagem autorregulada serão alimentadas atendendo às fontes de autoeficácia propostas por Bandura (1997). Entre essas fontes, a mais decisiva são experiências de êxito em tarefas anteriores, asseguradas com a adoção de estratégias eficazes. Estudos como o Baars e Wijnia (2018) e Müller e Seufert (2018) apresentaram evidências desse efeito sobre a autoeficácia. Resumidamente, alunos que, com algum tipo e grau de motivação inicial, usem ou tenham usado boas estratégias, previsivelmente, terão êxito e esse êxito constatado sinaliza para que esses alunos se sintam capazes de usar estratégias em novas tarefas, ou seja, com autoeficácia alentada. 
Em segundo lugar, os estudiosos da Teoria de Metas de Realização propuseram que a orientação duradoura à meta domínio será promovida, basicamente, pela estruturação psicológica da sala de aula, que inclui apresentação de tarefas desafiadoras, percebidas como significativas, formas de avaliação isentas de comparação social e o reconhecimento do esforço e uso de estratégias (para uma descrição mais detalhada, ver, por ex., GUIMARÃES, 2018). Já os autores da Teoria da Autodeterminação (RYAN; DECI, 2017) defendem que as formas mais autodeterminadas da motivação (motivações identificada e intrínseca, que compõem a autônoma) serão fomentadas quando o ambiente social atender às três necessidades básicas do ser humano - autonomia, competência e relacionamento. Em particular, a necessidade de competência será atendida quando professores apresentarem tarefas desafiadoras em nível que não exceda demasiadamente as competências atuais de seu aluno. Somente desafios em grau moderado, isto é, difíceis, mas acessíveis, além de estimularem a aplicação de esforço com estratégias, manterão e alimentarão o senso de competência em função do êxito conquistado (GUIMARÃES, 2018), uma relação similar ao que ocorre com a autoeficácia.

\section{Referências}

BAARS, M.; WIJNIA, L. The relation between task-specific motivational profiles and training of self-regulated learning skills. Learning and Individual Differences, Amsterdã, v.64, mayo 2018.

BANDURA, A. Self-efficacy, the exercise of control. New York: Freeman, 1997.

BERGER, J. L; KARABENICK, S. A. Motivation and students' use of learning strategies: evidence of unidirectional influences in mathematics classrooms. Learning and Instruction, Oxford, v. 21, n. 4, p. 416-428, 2011.

BOEKAERTS, M.; PINTRICH, P. R.; ZEIDNER, M. Handbook of self-regulation: theory, research, and applications. San Diego, CA: Academic Press, 2000.

BRADLEY, R. L.; BROWNE, B. L.; KELLEY, H. M. Examining the influence of self-efficacy and self-regulation in online learning. College Student Journal, Chula Vista, v. 51, n. 4, p. 518- 530, 2017.

BZUNECK, J. A. Emoções acadêmicas, autorregulação e seu impacto sobre motivação e aprendizagem. ETD - Educação Temática Digital, São Paulo, v. 20, n. 4, p. 1059-1075, out./dez. 2018. 
BZUNECK, J. A.; BORUCHOVITCH, E. Motivação e autorregulação da motivação no contexto educativo. Psicologia: Ensino \& Formação, Brasília, v. 7, n. 2, p. 73-84, 2016.

CAPRARA, G. V.; FIDA, R.; VECCHIONE, M.; DEL BOVE, G.; VECCHIO, G. M.; BARBARANELLI, C.; BANDURA, A. Longitudinal analysis of the role of perceived selfefficacy for self-regulated learning in academic continuance and achievement. Journal of Educational Psychology, Arlington, v. 100, n. 3, p. 525-534, 2008. DOI:10.1037/00220663.100.3.525.

DECI, E. L.; RYAN, R. M. Intrinsic motivation and self-determination in human behavior. New York: Plenum, 1985.

DONCHE, V.; MAEYER, S.; COERTJENS, L.; DAAL, T.; PETEGEM, P. Differential use of learning strategies in first-year higher education: the impact of personality, academic motivation, and teaching strategies. British Journal of Educational Psychology, Leicester, v. 83, n. 2, p. 238-251, 2013.

DÖRRENBÄCHER, L.; PERELS, F. Self-regulated learning profiles in college students: their relationship to achievement, personality, and the effectiveness of an intervention to foster self-regulated learning. Learning and Individual Differences, Greenwich, v. 51, p. 229-241, 2016.

GUAY, F.; CHANAL, J.; RATELLE,C. F.; MARSH, H. W.; LAROSE, S.; BOIVIN, M. V. Intrinsic, identified, and controlled types of motivation for school subjects in young elementary school children. British Journal of Educational Psychology, Leicester, v. 80, p. 711-735, 2010.

GUIMARÃES, S. E. R. A organização da escola e da sala de aula como determinante da motivação intrínseca e da meta aprender. In: BORUCHOVITCH, E.; BZUNECK, J. A. (org.). A motivação do aluno: contribuições da Psicologia contemporânea. Petrópolis: Editora Vozes, 2018. p. 78-95.

HULLEMAN, C. S.; SCHRAGER, S. M.; BODMANN, S. M.; HARACKIEWICZ, J. M. A metaanalytic review of achievement goal measures: different labels for the same constructs or different constructs with similar labels? Psychological Bulletin, Washington, v. 136, p. 422-449, 2010.

LEE, W.; LEE, M.; BONG, M. Testing interest and self-efficacy as predictors of academic self-regulation and achievement. Contemporary Educational Psychology, San Diego, v. 39, n. 2, p. 86-99, 2014.

LINNENBRINK-GARCIA, L.; WORMINGTON, S. V.; SNYDER, K. E.; RIGGSBEE, J.; PEREZ, T.; BEN-ELIYAHU, A.; HILL, N. Multiple pathways to success: an examination of integrative motivational profiles among upper elementary and college students. Journal of Educational Psychology, Arlington, v. 110, n. 7, p. 1026-1048, 2018.

LIU, W. C.; WANG, C. K. J.; KEE, Y. H., KOH, C.; LIM, B. S. C.; CHUA, L. College students' motivation and learning strategies profiles and academic achievement: a self- 
determination theory approach. Educational Psychology, Dorchester on Thames, v. 34, n. 3, p. 338-353, 2014.

MAEHR, M. L.; MEYER, H. A. Understanding motivation and schooling: we'v been, where we are, and where we need to go. Educational Psychology Review, New York, n. 9, p. 371-409, 1997.

MATOS, L.; LENS, W.; VANSTEENKISTE, M.; MOURATIDIS, A. Optimal motivation in Peruvian high schools: should learners pursue and teachers promote mastery goals, performance-approach goals or both? Learning and Individual Differences, Greenwich, v. 55, p. 87-96, 2017.

MCCOMBS, B. L. Historical review of learning strategies research: strategies for the whole learner-a tribute to claire ellen weinstein and early researchers of this topic. Frontiers in Education, New Delhi, v. 2, n. 6, p. 1-21, 2017.

MOURATIDIS, A.; MICHOUB, A.; DEMIRCIOĞUC, A. N.; SAYILD, M. Different goals, different pathways to success: performance-approach goals as direct and masteryapproach goals as indirect predictors of grades in mathematics. Learning and Individual Differences, Greenwich,v. 161, p. 127-135, 2018.

MÜLLER, N.; SEUFERT, T. Effects of self-regulation prompts in hypermedia learning on learning performance and self-efficacy. Learning and Instruction, Oxford, v. 58, p. 1-11, 2018.

NASCIMENTO, F. P. et al. Autorregulação e motivação em uma tarefa específica: uma abordagem centrada na pessoa. Estudos de Psicologia Campinas, Campinas, [2018]. No prelo.

PANADERO, E. A review of self-regulated learning: six models and four directions for research. Frontiers in Psychology, New Delhi, v. 8, p. 1-28, apr. 2017.

PAULINO, P.; SÁ, I.; SILVA, A. L. Autorregulação da motivação: crenças e estratégias de alunos portugueses do $7^{\circ}$ ao $9^{\circ}$ ano de escolaridade. Psicologia: Reflexão e Crítica, Porto Alegre, v. 28, n. 3, p. 574-582, 2015.

PINTRICH, P. R. The dynamic interplay of student motivation and cognition in the college classroom. In: AMES, C.; MAEHR, M. (ed.). Advances in motivation and achievement: motivation enhancing environments. Greenwich, CT: JAI Press, 1989. v. 6, p. 117-160.

PINTRICH, P. R. The role of goal orientation in self-regulated learning. In: BOEKAETS, M.; PINTRICH, P. R.; ZEIDNER, M. (ed.). Handbook of Self-Regulation:theory, research, and applications. San Diego, CA: Academic Press, 2000. p. 451-502. 
PINTRICH, P.R. A motivational science perspective on the role of student motivation in learning and teaching contexts. Journal of Educational Psychology, Arlington, v. 95, p. 667-686, 2003.

PINTRICH, P. R. A Conceptual framework for assessing motivation and self-regulated learning in college students. Educational Psychology Review, New York, v. 16, n. 4, p. 385-406, 2004.

RYAN, R. M.; DECI, E. L. Self-Determination theory: basic psychological needs in motivation, development, and wellness. New York: Guilford Press, 2017.

SCHUNK, D. H.; MEECE, J. L.; PINTRICH, P. R. Motivation in education: theory, research, and applications. 4. ed. Boston: Mass, 2014.

SCHUNK, D. H.; ZIMMERMAN, B. Motivation and self-regulated learning: theory, research, and applications. New York: Taylor \& Francis, 2008.

SENKO, C.; HULLEMAN, C. S. The role of goal attainment expectancies in achievement goal pursuit. Journal of Educational Psychology, Arlington, v. 105, n. 2, p. 504-521, 2013.

URDAN, T. Examining the relations among early adolescent students' goals and friends' orientation toward effort and achievement in school. Contemporary Educational Psychology, San Diego, v. 22, n. 2, p. 165-191, 1997.

USHER, E. L.; PAJARES, F. Self-efficacy for self-regulated learning: a validation study. Educational and Psychological Measurement, Durham, v. 68, p. 443-463, 2008.

VALLE, A.; CABANACH, R. G.; GONZÁLEZ-PIENDA, J. A.; NÚÑEZ, J. C.; RODRÍGUEZ, S.; ROSÁRIO, P. Perfiles motivacionales en estudiantes de secundaria: análisis diferencial en estrategias cognitivas, estrategias de autorregulación y rendimiento académico. Revista Mexicana de Psicología, Guadalajara, v. 26, n. 1, p. 113-124, 2009.

VANSTEENKISTE, M.; MOURATIDIS, A. Emerging trends and future directions for the field of motivation psychology: a special issue in honor of prof. dr. Willy Lens. Psychologica Belgica, Louvain, v. 56, n. 3, p. 118-142, 2016.

VANSTEENKISTE, M.; SIERENS, E.; SOENENS, B.; LUYCKX, K.; LENS, W. Motivational profiles from a self-determination perspective: the quality of motivation matters. Journal of Educational Psychology, Arlington, v. 101, p. 671-688, 2009.

WEINSTEIN, C. E.; MAYER, R. E. The teaching of learning strategies. In: Wittrock, M. C. (ed.). Handbook of research on teaching. New York: McMillan Publ. Co., 1986. p. 315327.

WEINSTEIN, C. E.; ACCE, T. W.; JUNG, J. Self-regulation and learning strategies. New Directions for Teaching and Learning, San Francisco, v. 126, p. 45-53, 2011. 
WOLTERS, C. A. Advancing achievement goal theory: using goal structures and goal orientations to predict students' motivation, cognition, and achievement. Journal of Educational Psychology, Arlington, v. 96, n. 2, p. 236-250, 2004.

ZIMMERMAN, B. J. Attaining self-regulation: a social cognitive perspective. In: BOEKAERTS, M.; PINTRICH, P. R.; ZEIDNER, M. (ed.). Handbook of self-regulation: theory, research, and applications. San Diego, CA: Academic Press, 2000. p. 13-39.

ZIMMERMAN, B. Investigating self-regulation and motivation: historical background, methodological developments, and future prospects. American Educational Research Journal, Washington, v. 45, n. 1, p. $166-183,2008$.

ZIMMERMAN, B. J. From cognitive modeling to self-regulation: a social cognitive career path. Educational Psychologist, Hillsdale, v. 48, n. 3, p.135-147, 2013.

ZIMMERMAN, B. J.; MARTINEZ-PONS, M. Student differences in self-regulated learning: relating grade, sex, and giftedness to self-efficacy and strategy use. Journal of Educational Psychology, Arlington, v. 82, p. 51-59, 1990.

ZUSHO, A. Toward an integrated model of [self-regulated] learning in the college classroom. Educational Psychology Review, New York, v. 29, p. 301-324, 2017. 